SHORT REPORT

\title{
Post-intervention effect of a computer tailored smoking cessation programme
}

\author{
Jean-François Etter, Thomas V Perneger
}

J Epidemiol Community Health 2004;58:849-851. doi: 10.1136/jech.2003.017103

$\mathrm{F}$ ace to face advice for smoking cessation is effective, but few smokers are willing to attend smoking cessation clinics, and many do not receive smoking cessation advice from their physician. Self help smoking cessation materials may not be very effective, ${ }^{2}$ but computer technology can be used to produce effective individualised self help smoking cessation materials and disseminate them widely at a low cost per participant. ${ }^{2}$ Most studies assessed the effect of computer tailored programmes on smoking cessation after one year or less. ${ }^{2}$ Only one programme was evaluated during 18 and 24 months. ${ }^{3}$ These programmes usually produced quit rates $<5 \%$ higher than in control groups who received standard self help materials or no intervention. ${ }^{2}$ Because over one third of ex-smokers who achieve 12 months of abstinence eventually relapse five years later, ${ }^{4}$ it is not yet known whether computer tailored programmes have a long term impact on smoking abstinence.

We showed previously that a computer tailored programme doubled the odds of quitting smoking, after seven months. ${ }^{5}$ In this study, we tested whether this effect was maintained 24 months after entry in the programme-that is, 12 months after the end of the intervention.

\section{METHODS}

In 1998, we sent the baseline questionnaire to a representative (random) sample of 20000 residents aged 18-60 in French speaking Switzerland. We sent out follow up questionnaires six and 23 months later to the 2934 baseline participants. We randomly assigned participants to the intervention or control group (fig 1).

The intervention consisted of personal counselling letters, written by a computer according to the answers made by participants to a 62 item questionnaire, and of stage matched brochures. Each counselling letter included about 20 paragraphs of text, and was compiled by the computer from a library of over 300 paragraphs. The content of the letters was based on extensive research conducted in the same population, ${ }^{5}$ and on relevant theory, in particular the transtheoretical model of behaviour change. ${ }^{3}$ The questionnaires used to produce the tailored counselling letters assessed the participants' demographic characteristics, stage of change ("precontemplation": no intention to quit smoking in the next six months, "contemplation": seriously considers quitting in the next six months, "preparation": has decided to quit in the next 30 days), ${ }^{3}$ level of tobacco dependence, perceived

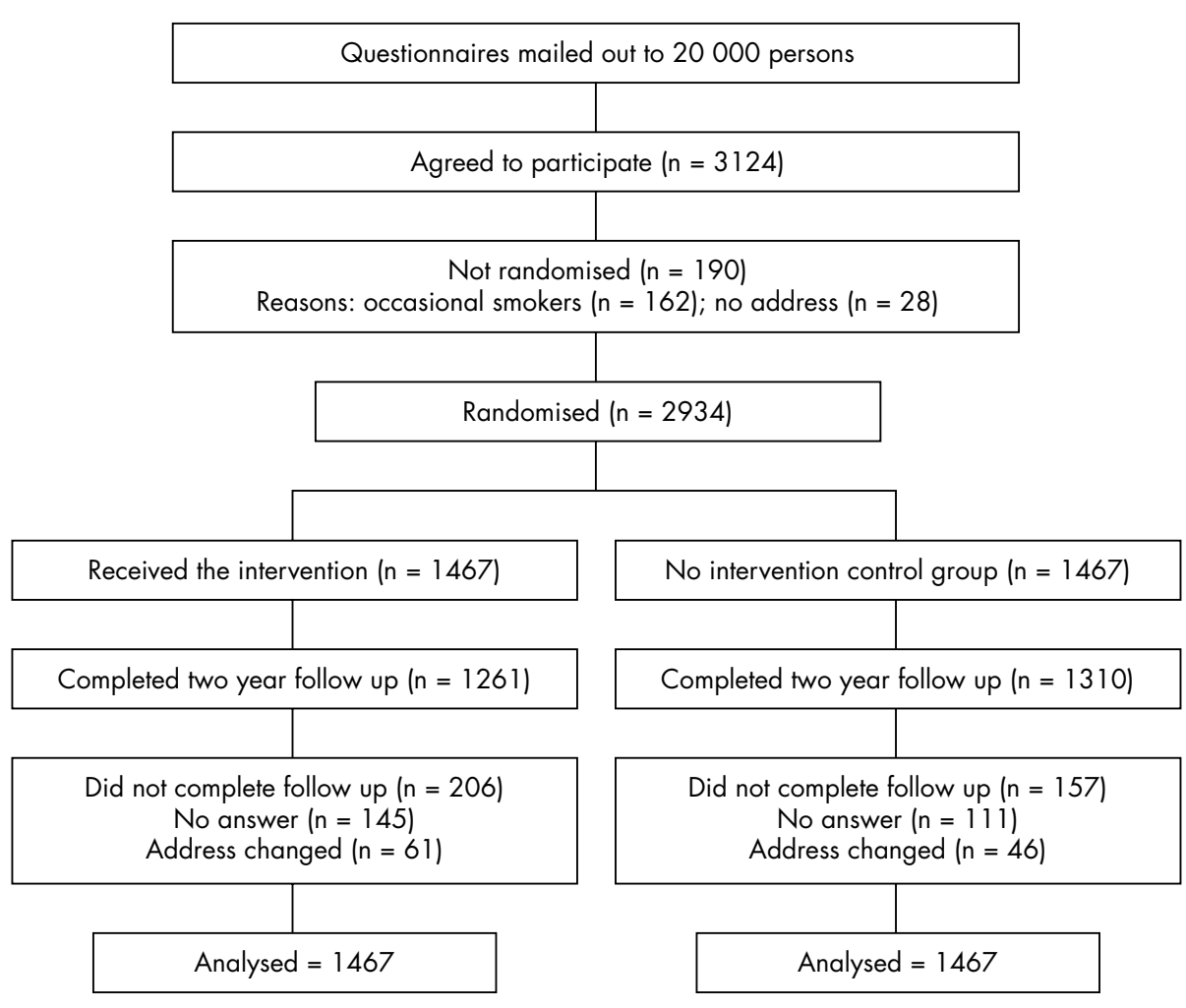

Figure 1 Flow chart of participants in the randomised controlled trial. 
Table 1 Smoking cessation rates 24 months after entry in the study, and 12 months after the end of the intervention. Intention to treat analysis. French speaking Switzerland, 19982001

\begin{tabular}{llll}
\hline & $\begin{array}{l}\text { Programme } \\
(\mathbf{n}=1467)\end{array}$ & $\begin{array}{l}\text { Control } \\
(\mathbf{n}=1467)\end{array}$ & p Value \\
\hline Four week smoking abstinence, number (\%) & $163(11.1)$ & $154(10.5)$ & 0.6 \\
Seven day smoking abstinence, number (\%) & $183(12.5)$ & $185(12.6)$ & 0.9 \\
Quit smoking for one month, during the study, number (\%) & $453(30.9)$ & $362(24.7)$ & $<0.001$ \\
Used $>$ 1 piece of a nicotine replacement product, number (\%) & $365(24.9)$ & $305(20.8)$ & 0.008 \\
Days without smoking, during the past two years (mean, SD) & $51.0(132)$ & $41.5(110)$ & 0.033 \\
Stage of change & $613(41.8)$ & $593(40.4)$ & 0.41 \\
Precontemplation & $576(39.3)$ & $611(41.6)$ & \\
Contemplation & $80(5.5)$ & $84(5.7)$ & \\
Preparation & $77(5.2)$ & $81(5.5)$ & \\
Action (that is, quit smoking less than six months ago) & $121(8.2)$ & $98(6.7)$ & \\
Maintenance (that is, quit smoking more than six months ago) & & & \\
\hline
\end{tabular}

drawbacks of smoking, self efficacy (that is, confidence in one's ability to refrain from smoking in specific situations), use of self change strategies (for example, obtaining support from family and friends), current use, and intention to use nicotine replacement therapy. We used validated scales to measure these variables. ${ }^{5}$ The counselling letters included cartoons and graphs, which were also tailored to the participants' characteristics. The questionnaire, counselling letters, and brochures can be seen at the Stop-tabac cessation programme web site (http://www.stop-tabac.ch).

After returning the baseline questionnaire, participants in the intervention group received by mail an eight page personal counselling letter and two 16 page booklets corresponding to their current stage of change and to the next stage. Two, four, and 12 months after entering the study, participants in the intervention group were invited to answer the tailoring questionnaire again, in order to receive a new counselling letter. Participants received counselling letters a median of two times over 12 months. Half the participants (45\%) received counselling only once, $40 \%$ twice, and $15 \%$ three times or more. Participants in the intervention group were not contacted between the 12th and the 24th months of the study.

After returning the baseline questionnaire, members of the control group received a letter indicating that they had been attributed to that group. They were not contacted again until the six month and 23 month follow up surveys.

The main outcome measure was one month abstinence (not having smoked even a puff of tobacco cigarette, cigar, pipe, etc, in the past four weeks), which is the criterion recommended by FDA. ${ }^{6}$ We also assessed one week abstinence (no puff of tobacco in the past seven days). We used an intention to treat analysis, in which all people absent at follow up were considered to be smokers, to have not used a nicotine replacement product, not made a quit attempt, not

\section{What this paper adds}

- Individualised computer tailored letters can be effective in helping smokers quit smoking, but it is not known whether this effect is maintained after the intervention is stopped.

- Even though a computer tailored programme doubled the odds of quitting smoking seven months after entry in the programme, this effect was not maintained one year after the intervention was stopped. modified their stage of change, and not spent any day without smoking.

We used $\chi^{2}$ tests to compare proportions and $t$ tests to compare means.

\section{RESULTS}

We recruited 2934 daily smokers at baseline. The participation rate to the first follow up was 84\% (2456 of 2934), seven months later. ${ }^{5}$ The participation rate to the second follow up was $88 \%$ (2571 of 2934), 24 months after baseline.

At baseline, participants were on average 36 years old, $48 \%$ were men, they had 14 years at school on average, they smoked on average 20 cigarettes per day and smoked their first cigarette on average 70 minutes after waking up, 40\% had made a quit attempt in the year before the study, $41 \%$ were in the precontemplation stage of change, $51 \%$ in the contemplation stage, and $8 \%$ in the preparation stage.

After seven months, the four week abstinence rates were $5.8 \%$ in the intervention group and $2.2 \%$ in the control group $(p<0.001)$, and the seven day quit rates were $8.0 \%$ and $3.3 \%$ $(\mathrm{p}<0.001)$, respectively. ${ }^{5}$ After 24 months, abstinence rates were similar in the two groups, but more participants in the intervention group than in the control group had made a one month quit attempt and had used nicotine replacement products during the study (table 1). The number of days of smoking abstinence during the study was higher in the intervention group than in the control group. After 24 months, the distribution of participants by "stage of change" was the same in the two groups.

Among participants who had quit smoking at seven month follow up, using the criterion of four weeks' abstinence, the proportions of non-smokers at 24 months were similar in the intervention and control groups (55.3\% versus 57.6\%, $\mathrm{p}=0.8$ ). Among smokers at seven months, the proportions of non-smokers at 24 months were similar in the two groups ( $8.4 \%$ versus $9.4 \%, \mathrm{p}=0.3$ ).

\section{DISCUSSION}

In daily smokers, a computer tailored smoking cessation programme carried out by mail doubled the odds of quitting smoking, seven months after entry in the programme, but this effect was not maintained after two years-that is, one

\section{Policy implications}

The post-intervention effect of available computer tailored smoking cessation programmes should be further tested, for example, two or five years after the intervention is stopped. 
year after the end of the intervention. This programme was effective only as long as it was active. This calls for an experimental test of various durations of the intervention (for example, one year compared with two or five years).

\section{ACKNOWLEDGEMENTS}

The smoking cessation programme described in this article is available free of charge on the internet and on paper. Vincent Baujard, from the Health On The Net Foundation (http://www.hon. ch) and Victor Gabriel developed the software for the smoking cessation programme.

\section{Authors' affiliations \\ J F Etter, T V Perneger, Institute of Social and Preventive Medicine, University of Geneva, Geneva, Switzerland \\ T V Perneger, Quality of Care Unit, Geneva University Hospitals, Geneva, Switzerland}

Funding: this study was supported by grants from the Swiss National Science Foundation to J-F Etter (32-47122-96, 3233-054994.98 and 3200-055141.98), by the Swiss Cancer League, the Swiss Federal Office of Public Health, and the Health Authority of the Canton of Geneva. The smoking cessation programme evaluated in this paper was funded by the same sponsors and by the Geneva Cancer League, the
Swiss Foundation for Health Promotion, the Loterie Romande, Pharmacia, and the Health Authority of the Canton of Jura.

Conflicts of interest: none declared.

Correspondence to: Mr J-F Etter, Institute of Social and Preventive Medicine, University of Geneva, CMU, case postale, CH-1211 Geneva 4, Switzerland; Jean-Francois.Etter@imsp.unige.ch

Accepted for publication 13 February 2004

\section{REFERENCES}

1 Silagy C, Stead LF. Physician advice for smoking cessation. Cochrane Library. Issue 1. Chichester, UK: Wiley, 2004

2 Lancaster T, Stead LF. Self-help interventions for smoking cessation. Cochrane Library. Issue 1. Chichester, UK: Wiley, 2004.

3 Prochaska JO, Velicer WF, Fava JL, et al. Evaluating a population-based approach and a stage-based expert system intervention for smoking cessation. Addict Behav 2001;26:583-602.

4 Blondal T, Gudmundsson $\mathrm{U}$, Olafsdottir I, et al. Nicotine nasal spray with nicotine patch for smoking cessation: randomised trial with six year follow up. BMJ 1999;30:285-8.

5 Etter JF, Perneger TV. Effectiveness of a computer-tailored smoking cessation program: a randomised trial. Arch Intern Med 2001;161:2596-601.

6 Food and Drug Administration. Transcript of the Joint Meeting of the Nonprescription Drugs Advisory Committee and the Drug Abuse Advisory Committee of the Food and Drug Administration. Rockville, MD: Food and Drug Administration, 1995.

\section{$\mathrm{ECHO}$}

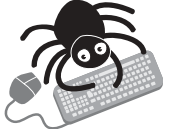

Please visit the Journal of Epidemiology and Community Health website [www.jech. com] for a link to the full text of this article.
Trends in hip and knee joint replacement: socioeconomic inequalities and projections of need

T Dixon, M Shaw, S Ebrahim, P Dieppe

Objectives: To examine trends in primary and revision joint (hip and knee) replacement in England between 1991 and 2000.

Methods: Analysis of hospital episodes statistics between 1 April 1991 and 30 March 2001 for total hip replacement (THR) and total knee replacement (TKR). Descriptive statistics and regression modelling were used to summarise patients' demographic and clinical characteristics and to explore variations in joint surgery rates by age, sex, and deprivation. Results: Between 1991 and 2000, the incidence of primary THR increased by $18 \%$, while the incidence of revision THR more than doubled. The incidence of primary TKR doubled, with revision TKR increasing by $300 \%$. Over the 10 year period, the proportion of THR episodes that involved revision operations rose from $8 \%$ to $20 \%$. Substantial variations in operation rates by socioeconomic status were seen, with the most deprived fifth of the population experiencing significantly lower rates. Projections estimate that primary THR numbers could rise by up to $22 \%$ by the year 2010 , with primary TKR numbers rising by up to $63 \%$.

Conclusions: Provision of joint replacement surgery in English NHS hospitals has increased substantially over the past decade. Revision operations in particular have increased markedly. The growth in primary operations has mostly occurred among those aged 60 years and over; rates among young people have changed very little. There is a significant deprivation based gradient in rates. If current trends continue there would be almost 47000 primary hip and 54000 primary knee operations annually by 2010 .

A Annals of the Rheumatic Diseases 2004;63:825-830. 